\title{
Intramedullary/intraosseous myelolipoma in a patient with pathologic fracture
}

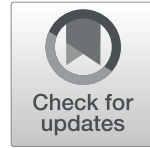

\author{
Anoshia Afzal', Sepideh N. Asadbeigi ${ }^{2^{*}} \mathbb{D}$, Maria Kamal', Lewis Hassell ${ }^{1}$ and Kar-Ming Fung ${ }^{1}$
}

\begin{abstract}
Background: Intramedullary lipomatous tumors are rare and mostly reported in the metaphysis of the femur, tibia, and fibula. Myelolipomas are very rare tumors composed of adipose tissue and hematopoietic cells commonly reported in adrenal gland. We report the first reported case of intraosseous myelolipoma with a pathologic fracture in a young woman.

Case presentation: Patient is a young woman who carried a diagnosis of systemic lupus erythematosus, antiphospholipid syndrome, and hyperparathyroidism. Radiologic studies were done after the patient reported right lower leg pain lasting for a month. Radiologic examination showed a large osteolytic lesions with pathologic fracture affecting the medial aspect of the proximal tibia with extensive soft tissue calcification. The initial clinical and radiographic suspicion was brown tumor secondary to the hyperparathyroidism. Curettage of the lesion yielded large fragments of mature adipose tissue punctuated by a variable amount of mature hematopoietic cells. The histopathologic features were suggestive of myelolipoma. The overall radiographic and histopathologic features supported a diagnosis of myelolipoma. The diagnosis of intraosseous myelolipoma can be challenging in small samples such as core biopsies.

Conclusions: The differential diagnoses of intraosseous myelolipoma include normal bone marrow, intramedullary hematopoiesis, and other benign lesions. The gold standard diagnosis is histopathologic examination. However, clinical and radiographic features have important roles in the diagnosis of this rare lesion. Due to the rarity of this tumor and lack of formal guidelines for management, the case-to-case basis treatment is recommended.
\end{abstract}

Keywords: Intraosseous, tibia, myelolipoma, pathologic fracture, lipoma

\section{Introduction}

Myelolipoma is an infrequent benign mesenchymal tumor that is comprised of mature adipose tissue mixed with hematopoietic components and is usually found in the adrenal gland. Myelolipomas have been described in extra-adrenal sites such as the stomach, liver, mediastinum and kidneys but are extremely rare inside the bone (Sawhney et al., 2006; Kenney et al., 1998; Wood et al., 2013; Jaewon et al., 2019). The absence of hematopoetic

\footnotetext{
* Correspondence: Sepideh.asadbeigi@northwestern.edu

Parts of this article were previously presented at the College of American Pathologists (CAP) 2020 Annual Meeting which was held from October 10, 2020 to October 14, 2020, and parts of the abstract were published in Archives (CAP Journal, September 2020 edition).

${ }^{2}$ McGaw Medical Center of Northwestern University, Chicago, IL, USA Full list of author information is available at the end of the article
}

elements differentiates myelolipomas from intraosseous lipomas. Myelolipoma is a rare entity and most frequently located in the adrenal glands (Plaut, 1958). Approximately 100 extra-adrenal myelolipomas (EAMLs) have been reported, most often in the abdomen, retroperitoneum, and mediastinum (Baker et al., 2012). Most patient are asymptomatic, and the tumor is nonfunctional. However, myelolipoma can grow in size and undergo hemorrhage and necrosis. Also, depending on the site and size of the lesion, myelolipomas can cause signs and symptoms of pressure on other parts of body. Pain and swelling are the most common symptoms when clinical manifestations present. Other locations are uncommon and mostly asymptomatic (Wen et al., 2015; Bokhari et al., 2020; Barman et al., 2014). Intra-osseous

(c) The Author(s). 2022 Open Access This article is licensed under a Creative Commons Attribution 4.0 International License, which permits use, sharing, adaptation, distribution and reproduction in any medium or format, as long as you give appropriate credit to the original author(s) and the source, provide a link to the Creative Commons licence, and indicate if changes were made. The images or other third party material in this article are included in the article's Creative Commons licence, unless indicated otherwise in a credit line to the material. If material is not included in the article's Creative Commons licence and your intended use is not permitted by statutory regulation or exceeds the permitted use, you will need to obtain permission directly from the copyright holder. To view a copy of this licence, visit http://creativecommons.org/licenses/by/4.0/. 
myelolipomas (IOML) are extremely rare with only 7 cases reported in the English and Non-English literature (Jaewon et al., 2019; Wen et al., 2015; Papapietro et al., 2009; Sundaram et al., 2007; Chiarini et al., 1992; Selye \& Stone, 1950; Sakai et al., 2021). Considering the very low incidence of this tumor in bone, it is rarely considered in the differential diagnosis of intraosseous tumors. Herein, we present a case of a primary IOML and will review the 7 previously reported cases in order to increase the awareness of diagnostic criteria of this rare tumor.

\section{Case Presentation}

We report a case of a 25 years old female with a complicated past medical history including longstanding systemic lupus erythematosus (SLE), lupus nephritis and associated end-stage renal disease (ESRD), secondary hyperparathyroidism, seizure, and deep venous thrombosis (DVT). The patient reported unilateral right leg pain for one month. Radiographs showed large lytic lesions throughout the proximal two third of left tibia with the largest lesions located on the anterior and proximal aspects. The right tibia also showed large lytic lesions involving the mid to distal fibula and the proximal $2 / 3$ of the tibia (Fig. 1), with pathologic fractures affecting the tibiae, bilaterally. The radiographs also showed extensive soft tissue and atherosclerotic calcification of the popliteal, anterior and posterior tibial arteries. The patient underwent bilateral open reduction and internal fixation (ORIF) by orthopedic surgery.
Histologically, curettage of the left tibial lesion yielded large fragments of mature adipose tissue populated by variable amounts of mature hematopoietic cells. No bone trabeculae were identified (Fig. 2). The overall radiographic and histopathologic features supported a diagnosis of IOML. The post-op laboratory work up showed vitamin D deficiency, and severe secondary hyperparathyroidism with parathyroid hormone $(\mathrm{PTH})$ levels of $4494 \mathrm{pg} / \mathrm{mL}$ (normal range $6.0-48.0 \mathrm{pg} / \mathrm{mL}$ ). Consequently, she underwent 3.5-gland parathyroidectomy with half-gland implantation into the neck, and this decreased the PTH level to $129.6 \mathrm{pg} / \mathrm{mL}$ in a 4-week follow up. To complete the work-up, a bone marrow biopsy was done and it was unremarkable except for a mild increase in iron stores. One month later, radiological studies showed a closed, displaced right supracondylar femur fracture which was reduced and internally fixed. No additional lesions compatible with myelolipoma were identified. The 6-month follow-up showed diffuse osseous demineralization with no fractures or tumoral lesions but there were mild degenerative changes throughout the foot along with marked vascular and soft tissue calcifications. The patient continued on her treatment for lupus, DVT and ESRD and has not had any sign of myelolipoma recurrence to date.

\section{Discussion}

Myelolipomas are relatively unusual, benign tumors comprised of a mixture of mature adipose and hematopoietic tissue most commonly found in adrenal

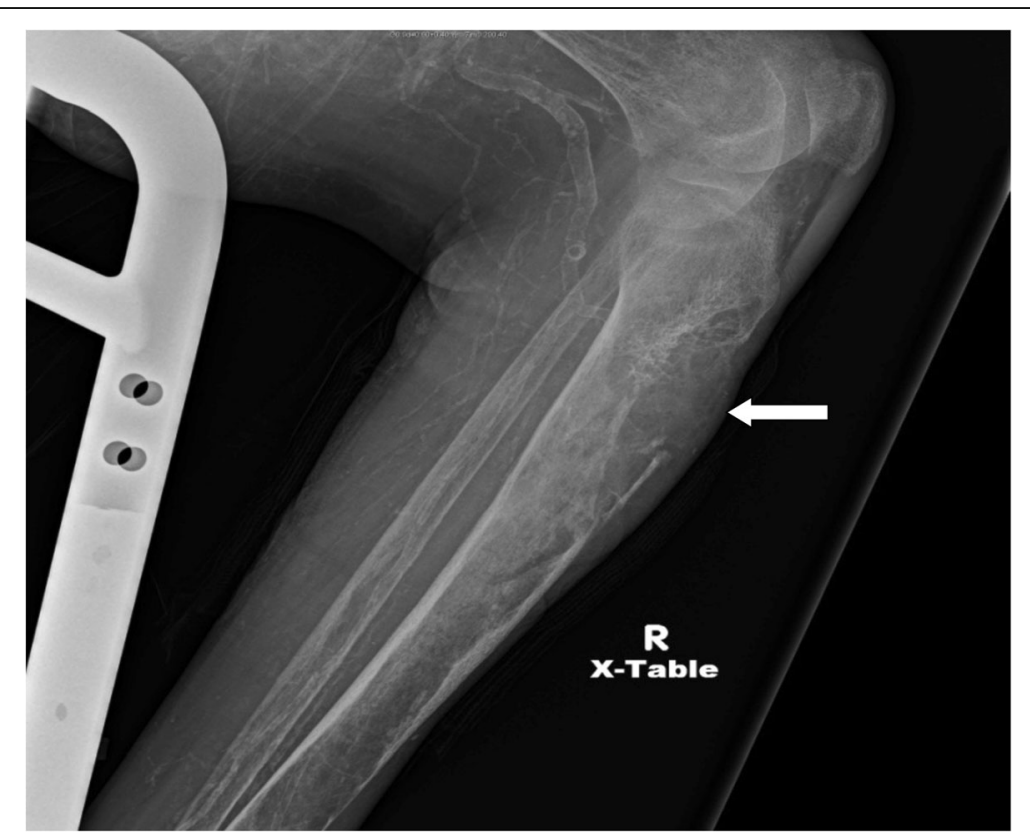

Fig. 1 Radiologic examination showed a large osteolytic lesion with pathologic fracture affecting the medial aspect of the proximal tibia with extensive soft tissue calcification 


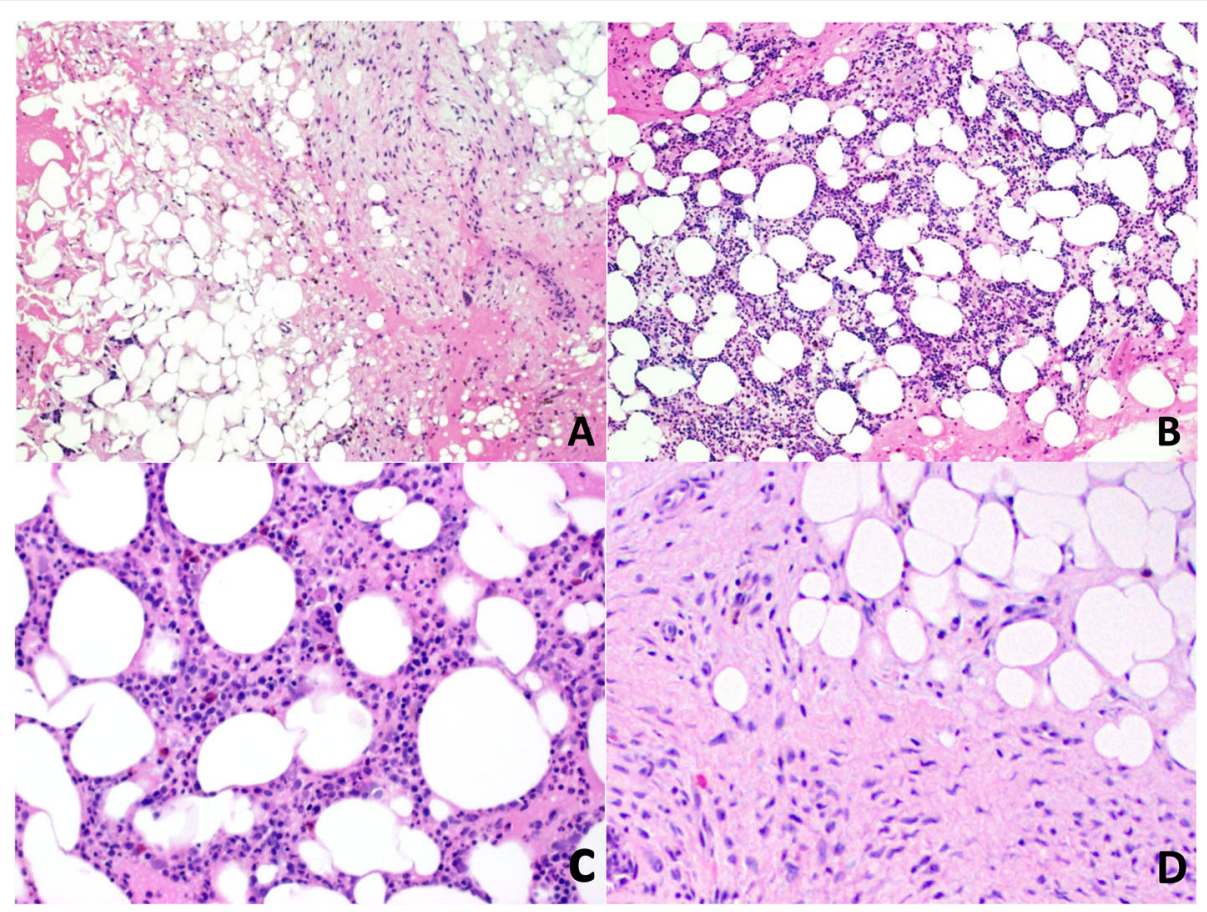

Fig. 2 Hematoxylin and Eosin staining (A: 4x10, B: 10×10, C \& D: 20x10) A: Curettage sections yielded fragments of mature adipose tissue. B, C: Variable amount of normocellular mature hematopoietic cells from all three lineages are present. D: Mature adipose and fibrotic tissue is present. No osteoclastic giant cells, hemorrhage or bony tissue was identified

glands (Sawhney et al., 2006; Kenney et al., 1998). EAMLs are rare, with only a handful of reported cases (Wood et al., 2013; Shen et al., 2014; Sagan et al., 2009; Fowler et al., 1982) and the retroperitoneum as the most common extra-adrenal location (Kenney et al., 1998). Our literature review reveals that IOML are extremely rare, with only seven cases reported in the period of 19922019 in English and non-English literature (Table 1) (Wen et al., 2015; Papapietro et al., 2009; Sundaram et al., 2007; Chiarini et al., 1992; Sakai et al., 2021).

Myelolipomas usually happen in late adulthood and have no gender predilection. The reported intra-osseous cases showed a variety of ages and no gender predilection (Bokhari et al., 2020). Even though most of myelolipomas are asymptomatic and may be incidental findings (Bokhari et al., 2020; Hakim \& Rozeik, 2014), all the reviewed IOML patients were symptomatic and the workups were initiated due to their signs or symptoms.

Several etiologies have been proposed for adrenal myelolipomas, including the degeneration of hyperplastic nodules or adrenal adenomas or myeloid and mesenchymal cell hyperplasia, which are erroneously displaced during embryogenesis. The literature also suggests that myelolipomas can result from stress-induced metaplasia of the reticuloendothelial tissue or prolonged stimulation of erythropoiesis in chronic anemia (Wilhelmus et al., 1981). The other possible proposed etiologies include seeded bone marrow emboli, metaplasia of adrenal stromal cells, or the metaplasia of reticuloendothelial cells (Merchant et al., 2002).

However, all proposed etiologies rely on only hypotheses due to the rarity of this tumor. Bishop et al. suggested a clonal origin for myelolipoma (Bishop et al., 2006). Seyle and his colleages were the first to produce myeloid tissue in the adrenal glands of rats by injecting necrotic tissue into those glands, and this suggested that hematopoetic metaplasia may be caused by hormonal stimulation of the adrenocortical tissue (Selye \& Stone, 1950).

Stressful events such as cancers, renal diseases, and cardiac lesions may also contribute to this transformation (Selye \& Stone, 1950). Feng et al. hypothesized that the stimulation of mesenchymal endothelial cells produce adipose tissue and that the adipocytes become inflammatory as they mature, which in turn stimulates the neighboring adrenal cortex which releases granulocyte colony-stimulating factor (G-CSF) which recruits the circulating hematopoietic progenitors (Feng et al., 2013). Overall, most of the authors accept the hypothesis that the metaplasia occurs in the reticuloendothelial cells of the blood capillaries after a stressful stimulus (Decmann et al., 2018). However, due to the extreme rarity of this tumor in bone, no hypothesis for the etiology of IOML has been suggested. In our patient, a long history of chronic autoimmune disease and secondary hyperparathyroidism could have triggered a stressful event which 


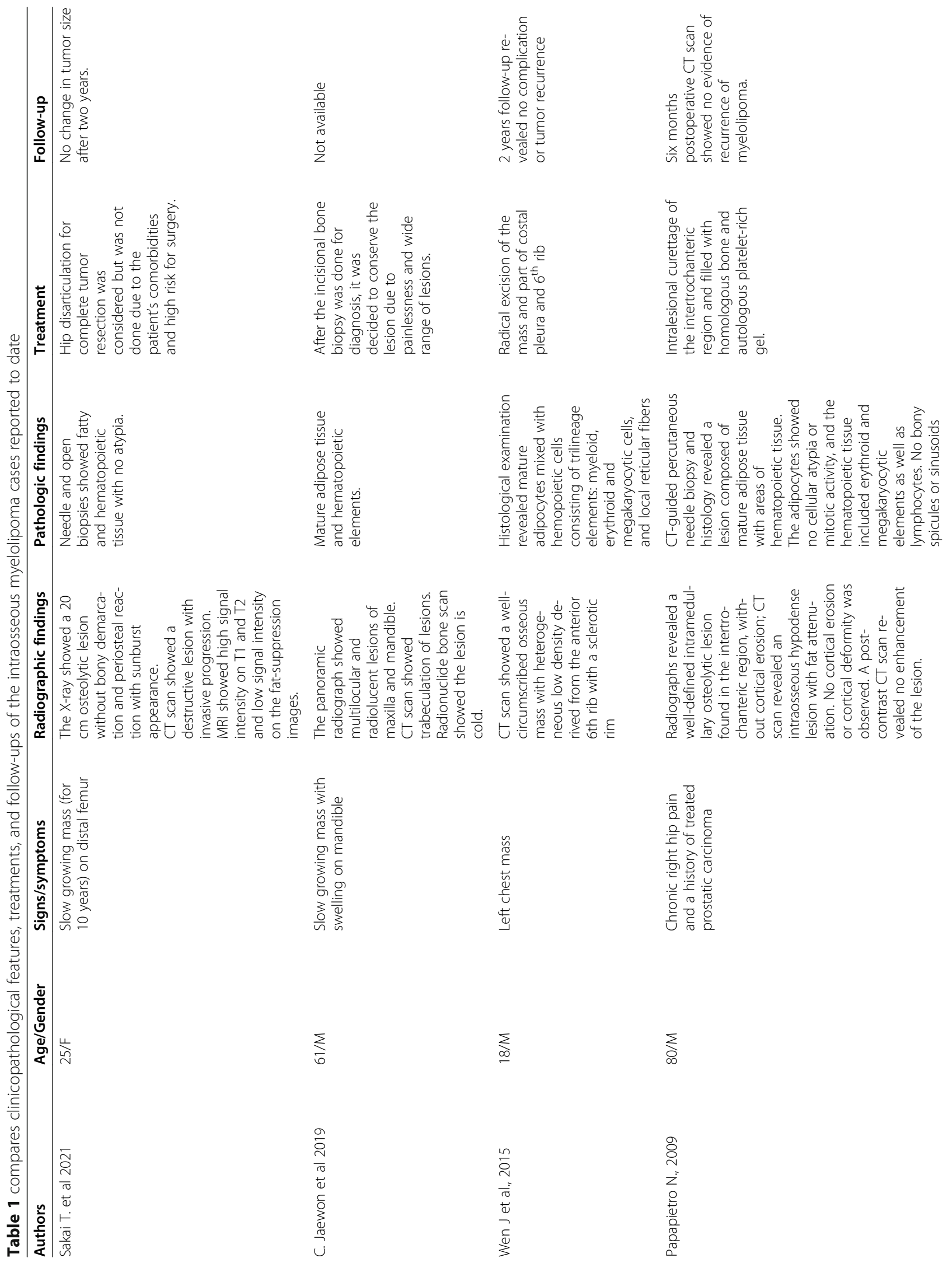




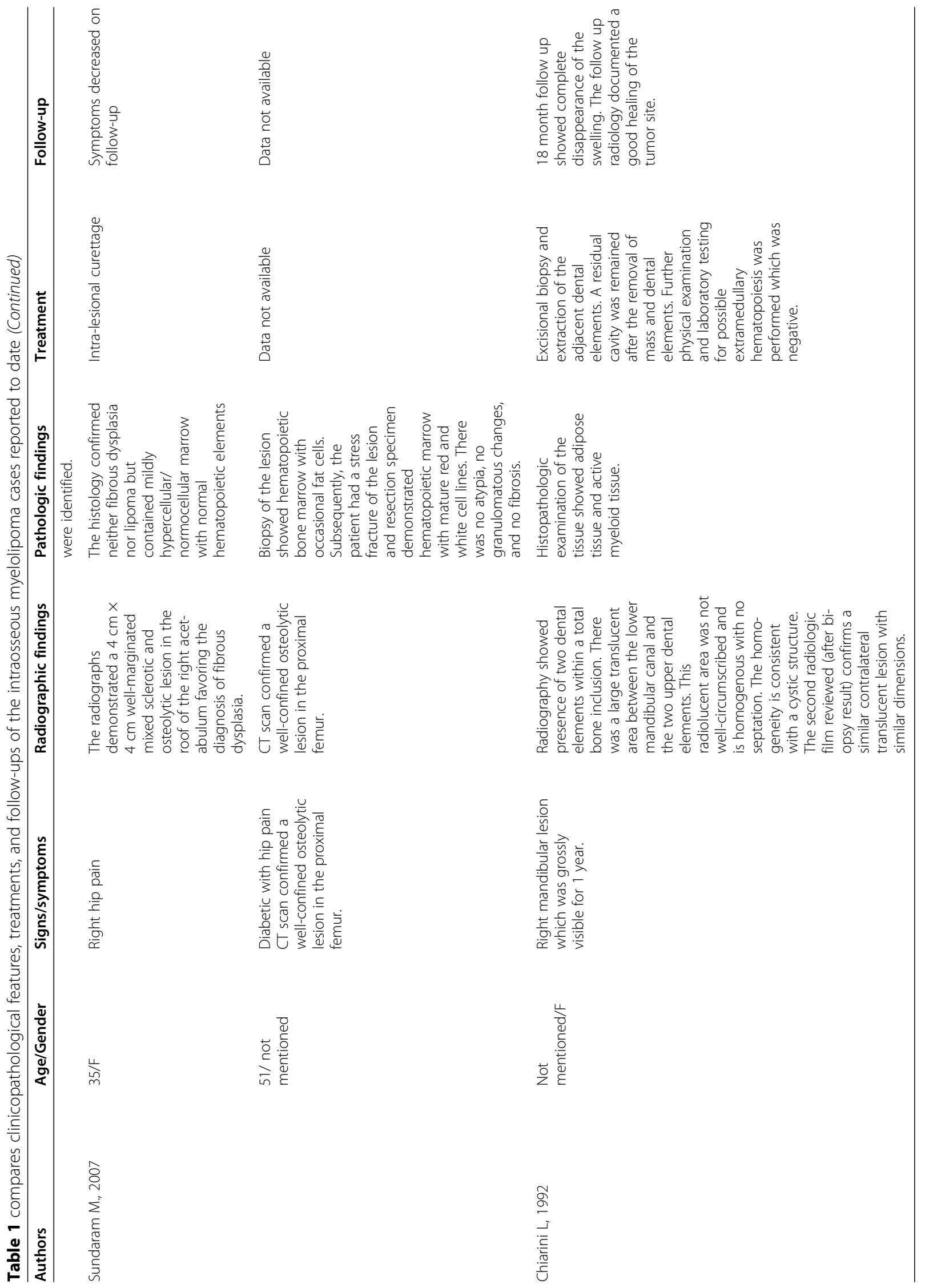


stimulated the release of G-CSF to alter mesenchymal cells in the large areas of osseous demineralization. Alternatively, both of the IOML cases with mandibular and maxillary involvement, the lesions were close to the teeth, were multifocal, and were more extensive than the other reported IOMLs, suggesting the possibility of mesenchymal origins. However, given the low number of reported cases and limited available data, we are still not able to go beyond few reported hypotheses in considering the etiology of IOML.

On radiology, myelolipomas usually show a hypodense mass that may have a heterogeneous or homogeneous appearance. Distinguishing myelolipoma from other fatcontaining tumors is difficult. Considering the different radiologic manifestations, it is nearly impossible to reach a definitive diagnosis without a histologic examination (Hakim \& Rozeik, 2014; Decmann et al., 2018). The radiologic finding in our case showed multiple lytic lesions with a pathologic fracture but the correlation of this fracture with myelolipoma is doubtful given that the patient has a history of hyperparathyroidism, ESRD, and vitamin $\mathrm{D}$ deficiency. She also developed an additional pathologic fracture after the removal of myelolipoma and we suspect myelolipoma is more of a co-morbidity rather than a causal factor. In the reported cases, a variety of radiological findings were present but all were consistent with a low density or osteolytic lesion ranging from a hypodense foci to a tumoral mass (Table 1). Most patients presented with a single intraosseous lesion with the exception of the two cases on the maxilla and mandible which presented with multiple, wide lesions (Jaewon et al., 2019; Chiarini et al., 1992). Sundaram et al. reported that the first reported Italian case of IOML did not have a pre-operative radiology study but in fact such a study was performed (Sundaram et al., 2007; Chiarini et al., 1992). Thus, all reported cases had radiological work-ups before the excisional or curettage procedures. Nevertheless, myelolipoma was confirmed only based on the histopathologic examination of the tissue.

To establish a diagnosis of myelolipoma, the presence of hematopoietic and adipose tissue should not have any justification in that organ and the formation of the myeloadipose tissue in the site should not be secondary to a physiologic hematopoietic hyperplastic response (Chiarini et al., 1992). When myelolipoma occurs in extra-osseous locations, a high suspicion for that diagnosis is raised by a needle biopsy followed by a pathological examination, which shows the mature adipose tissue with trilineage hematopoietic elements (Bokhari et al., 2020; Yang et al., 1992). However, in IOML, diagnosis is further challenged by the presence of adipose tissue as a part of the bone marrow and the seldom presence of hematopoietic cells mixed with adipocytes. The differential diagnosis for an intra-osseous tumor-like lesion is vast and heavily dependent on location, age, and radiologic findings. Myelolipomas are composed of both mature fat and hematopoietic elements and the diagnosis is made based on ruling out the other histopathological counterparts (Sundaram et al., 2007). The biopsy findings can resemble focal hematopoietic hyperplasia (FHH) which is histologically known to show hyperplastic marrow elements merging with fatty marrow with no hematopoietic dyspoiesis or malignancy (Wen et al., 2015; Galindo et al., 1998). In contrast, myelolipomas show normal cellularity. All reported IOML cases showed marrow cellularity within the normal range for the patient's age. Intra-osseous lipomas are one of the rarest bone tumors and while they mostly occur in the lower limbs, they can occur in any bone. The histologic examination of these tumors can show different stages of lipocyte involution but generally do not show any hematopoietic cells (Milgram \& Intraosseous lipomas., 1988). Other differential diagnoses to consider include marrow hyperplasia in response to chronic hemolysis or excessive usage of blood cells due to an underlying disease. However, anemia and hepatosplenomegaly are usually associated with these cases. Hyperplasia of bone marrow can be seen in adults with no chronic hemolysis (Shellock et al., 1992). This pattern of hyperplasia shows the dominancy of the red component of bone marrow in contrast to myelolipoma, which usually shows the normal proportion of hematopoietic lineages among the different lineages.

To date, the reported locations of myelolipoma include humerus, acetabulum, femur, rib, mandible, and maxilla. We report the first case of tibial myelolipoma accompanied with a pathologic fracture. The follow up of our patient did not show any evidence of myelolipoma recurrence but did show multiple fibular fractures which led the clinician to remove the parathyroid glands in order to prevent future pathologic fractures.

IOML is managed conservatively or surgically depending on the diagnostic certainty, the location of the lesion, and the disease progression. Due to the rare incidence of IOML, the existing literature contains no treatment guidelines. In all the cases where follow-up data was available, none of the patients showed recurrence or a malignant transformation.

\section{Conclusion}

IOML is a benign and extremely rare tumor composed of adipose and hematopoietic tissue. The first case report of an intra-osseus myelolipoma is from an Italian dentistry journal in 1992 which was either misinterpreted or not further studied due to the unavailability of an English translation. We are presenting the largest literature review of IOML in both the English and nonEnglish literature. Due to the lack of formal guidelines 
for managing IOMLs, the case-to-case basis treatment has been recommended. It appears to us that surgical excision or curetting are necessary for diagnostic purposes and may reduce the mass effect or the associated symptoms. The risk of recurrence after excision is minimal and no malignant transformation has been reported. However, more case reports are needed to specify the characteristics of this lesion and develop more consistent and certain diagnostic criteria with treatment protocols.

\section{Abbreviations \\ EAML: Extra-adrenal myelolipomas; IOML: Intra-osseous myelolipomas; SLE: Systemic lupus erythematosus; ESRD: End-stage renal disease; ORIF: Open reduction and internal fixation; PTH: Parathyroid hormone; G- CSF: Granulocyte colony-stimulating factor; FHH: Focal hematopoietic hyperplasia}

\section{Acknowledgements}

None

\section{Authors' contributions}

AA gathered the data and was a major contributor in writing the manuscript. SNA reviewed the existing literature and was a major contributor un the writing the manuscript. MK helped with writing the manuscript. LH performed the histological examination of the tissue and edited the manuscript. KMF was the senior author and made did the histologic examination, made the final diagnosis was a major contributor in writing the manuscript. All authors read and approved the final manuscript.

\section{Funding}

None

\section{Availability of data and materials}

Not applicable

\section{Declarations}

\section{Ethics approval and consent to participate}

OUHSC does not require IRB for case reports with no identification information.

\section{Consent for publication}

Not applicable. No identifiable information/ clinical picture is provided in the manuscript.

\section{Competing interests}

None

\section{Author details}

${ }^{1}$ University of Oklahoma Health Sciences Center, Oklahoma City, OK, USA.

${ }^{2}$ McGaw Medical Center of Northwestern University, Chicago, IL, USA.

Received: 25 July 2021 Accepted: 23 November 2021

Published online: 28 January 2022

\section{References}

Baker KS, Lee D, Huang M, Gould ES. Presacral myelolipoma: a case report and review of imaging findings. J Radiol Case Rep. 2012;6(6):1-9.

Barman S, Mandal KC, Mukhopadhyay M. Adrenal myelolipoma: An incidental and rare benign tumor in children. J Indian Assoc Pediatr Surg. 2014;19(4): 236-8.

Bishop E, Eble JN, Cheng L, et al. Adrenal myelolipomas show nonrandom Xchromosome inactivation in hematopoietic elements and fat: support for a clonal origin of myelolipomas. Am J Surg Pathol. 2006;30(7):838-43.

Bokhari MR, Zulfigar H, Garla W. Adrenal Myelolipoma. Treasure Island (FL): StatPearls; 2020.
Chiarini L, Bertoldi C, Criscuolo M, Ferronato G. Myelolipomatosis. A report of a case located in the mandible. Minerva Stomatol. 1992;41(4):165-72.

Decmann A, Perge P, Toth M, Igaz P. Adrenal myelolipoma: a comprehensive review. Endocrine. 2018;59(1):7-15.

Feng $\mathrm{C}$, Jiang $\mathrm{H}$, Ding $\mathrm{Q}$, Wen $\mathrm{H}$. Adrenal myelolipoma: a mingle of progenitor cells? Med Hypotheses. 2013;80(6):819-22.

Fowler MR, Williams RB, Alba JM, Byrd CR. Extra-adrenal myelolipomas compared with extramedullary hematopoietic tumors: a case of presacral myelolipoma. Am J Surg Pathol. 1982;6(4):363-74.

Galindo LM, Soans S, Chiaramonte C, Garcia FU. Focal hematopoietic hyperplasia of the rib. Report of a case diagnosed by fine needle aspiration. Acta Cytol. 1998;42(4):987-90.

Hakim A, Rozeik C. Adrenal and extra-adrenal myelolipomas - a comparative case report. J Radiol Case Rep. 2014;8(1):1-12.

Jaewon C, Paeng JY, Ahn J. Intraosseous Myelolipoma of Maxilla and Mandible. Korean J Oral Maxillofacial Pathol. 2019;43:217-21.

Kenney PJ, Wagner BJ, Rao P, Heffess CS. Myelolipoma: CT and pathologic features. Radiology. 1998;208(1):87-95.

Merchant SH, Herman CM, Amin MB, Ro JY, Troncoso P. Myelolipoma associated with adrenal ganglioneuroma. Arch Pathol Lab Med. 2002;126(6):736-7.

Milgram JW, Intraosseous lipomas. A clinicopathologic study of 66 cases. Clin Orthop Relat Res. 1988;231:277-302.

Papapietro N, Martinelli N, Ippolito M, Amato C, Denaro V. Intraosseous myelolipoma within proximal femoral metaphysis in a patient with previous prostate cancer: a case report. Hip Int. 2009;19(3):283-6.

Plaut A. Myelolipoma in the adrenal cortex; myeloadipose structures. Am J Pathol. 1958;34(3):487-515.

Sagan D, Zdunek M, Korobowicz E. Primary myelolipoma of the chest wall. Ann Thorac Surg. 2009;88(4):e39-41.

Sakai T, Aiba H, Nakagawa M, Hattori H, Murakami H, Kimura H. Myelolipoma mimicking osteosarcoma in the distal femur. Int I Surg Case Rep. 2021;83: 105997.

Sawhney R, McRae B, Lazarchick J. A rare case of a multifocal extra-adrenal myelolipoma with markedly hypocellular bone marrow. Ann Clin Lab Sci. 2006:36(2):208-11.

Selye $\mathrm{H}$, Stone $\mathrm{H}$. Hormonally induced transformation of adrenal into myeloid tissue. Am J Pathol. 1950;26(2):211-33.

Shellock FG, Morris E, Deutsch AL, Mink JH, Kerr R, Boden SD. Hematopoietic bone marrow hyperplasia: high prevalence on MR images of the knee in asymptomatic marathon runners. AJR Am J Roentgenol. 1992;158(2):335-8.

Shen C, Han Z, Che G. A bilateral neoplasm in chest: a case report and literature review. BMC Surg. 2014;14:42.

Sundaram M, Bauer T, von Hochstetter A, llaslan H, Joyce M. Intraosseous myelolipoma. Skeletal Radiol. 2007;36(12):1181-4.

Wen J, Chen H, Yi W, Zhou X. Primary costal myelolipoma: a case report and review of the literature. Int J Clin Exp Pathol. 2015;8(2):2202-4.

Wilhelmus JL, Schrodt GR, Alberhasky MT, Alcorn MO. Giant adrenal myelolipoma: case report and review of the literature. Arch Pathol Lab Med. 1981;105(10): $532-5$.

Wood WG, Restivo TE, Axelsson KL, Svahn JD. Myelolipoma in the spleen: a rare discovery of extra-adrenal hematopoietic tissue. J Surg Case Rep. 2013; 2013(3).

Yang GC, Coleman B, Daly JM, Gupta PK. Presacral myelolipoma. Report of a case with fine needle aspiration cytology and immunohistochemical and histochemical studies. Acta Cytol. 1992;36(6):932-6.

\section{Publisher's Note}

Springer Nature remains neutral with regard to jurisdictional claims in published maps and institutional affiliations. 